\title{
Special Issue on “Computational Methods for Polymers"
}

\author{
Masoud Soroush $\mathbb{D}$ \\ Department of Chemical and Biological Engineering, Drexel University, Philadelphia, PA 19104, USA; \\ soroushm@drexel.edu
}

Received: 17 March 2020; Accepted: 17 March 2020; Published: 26 March 2020

check for updates

Polymers play a key role in our daily lives. Natural polymers include proteins, cellulose, nucleic acids, lignin, natural rubber, and wood resins. Examples of manmade polymers are synthetic rubbers and plastics. To describe polymer properties, processes and reactions mathematically, phenomena at different length and time scales should be accounted for. The length scales are from angstrom to meter, and the time scales are from femtosecond to hour. The wide ranges of the scales require the use of an appropriate modeling method or a combination of appropriate methods. Examples of the methods are: quantum chemical/mechanical methods such as density functional theory (electronic scale); molecular dynamics and Monte Carlo (molecular scale); dissipative particle dynamics, Brownian dynamics, and lattice Boltzmann method (microscopic scale); dissipative particle dynamics and field theoretic polymer simulation (meso scale); and control volume methods and finite element (macroscopic scale) [1-3].

This Special Issue on "Computational Methods for Polymers" includes five articles on the mathematical modeling of polymers, polymer processes and polymerization reactions. The methods used include Monte Carlo simulations, macroscopic-scale modeling, and electronic-scale modeling. These articles are then followed by three on state and parameter estimation, one on model-based control of a molding process, and one on the operability analysis of a polymer membrane reactor. Next, there are three articles on macromolecular structural properties. The Special Issue then ends with two articles, one on the impact, spreading and rebound of a droplet, and the other on the impacts of branching and backbiting reactions on pulsed laser polymerization (PLP).

The first article is on how to produce hyperbranched (HB) polymer architectures in reactors. Tobita [4] studied the fundamental structural characteristics of polymer chains formed in representative types of reactors. In particular, he investigated irreversible step growth polymerization of an AB2 type monomer in a batch and a continuous stirred-tank reactor (CSTR) via Monte Carlo simulations. The simulation results revealed that in a CSTR, a highly branched core region consisting of units with large residence times is formed to give much more compact architecture compared to batch polymerization.

Atan et al. [5] review mathematical models developed for olefin polymerization processes. Their review covers coordination and free-radical mechanisms in different types of reactors such as fluidized bed, horizontal-stirred-bed, vertical-stirred-bed, and tubular. They present guidelines for the mathematical modeling of gas-phase olefin polymerization processes.

Riazi et al. [6] consider more than 40 reactions that are most likely to occur in high-temperature free-radical homopolymerization, and derive moment rate equations for the reactions methodically. Using a step-by-step approach based on the method of moments, their article guides the reader to determine the contributions of each reaction to the production or consumption of each species as well as to the zeroth, first and second moments of chain-length distributions of live and dead polymer chains, in order to derive the overall rate equation for each species, and to derive the rate equations for the leading moments of different chain-length distributions.

Zaccaria et al. [7] used electronic-scale modeling (density functional theory [DFT]) to study four $\mathrm{Cl} / \mathrm{Me}$ substituted [ONNO] Zr-catalysts for ethene/ $\alpha$-olefin polymerization. They found that replacing 
electron-donating methyl with isosteric but electron-withdrawing chlorine substituents results in a significant increase in comonomer incorporation. Using DFT, they explored the steric and electronic properties of the ancillary ligand and found that the relative reactivity ratios are mainly determined by the electrophilicity of the metal center. They report that electronic effects observed in these catalysts affect the relative barriers for insertion and a capture-like transition state.

Darvishi et al. [8] use electronic-scale modeling to study undesired polymerization of styrene during distillation, storage, and transportation. They apply DFT and carried out laboratory experiments to study the antipolymer and antioxidant activity of stable nitroxide radicals and phenolics in styrene polymerization. They report that 2,6-di-tert-butyl-4-methoxyphenol, 2,6-di-tert-butyl-4-methylphenol, 4-hydroxy-2,2,6,6-tetramethyl piperidine 1-Oxyl, and 4-oxo-2,2,6,6-tetramethylpiperidine 1-Oxyl are the most effective inhibitors for styrene.

Lathrop et al. [9] report an experimental and theoretical/computational study on modeling and monitoring reversible addition-fragmentation chain-transfer (RAFT) polymerization of methyl methacrylate. They carry out parameter estimation to determine RAFT reaction rate coefficients and an initiator efficiency from in-situ ${ }^{1} \mathrm{H}$ NMR and SEC experimental data. Based on their model, they implement a multi-rate multi-delay observer that include an inter-sample predictor and a deadtime compensator. Their results show that the multi-rate multi-delay observer has satisfactory convergence after a few sampling periods.

Salas et al. [10] present a data-driven strategy for the online estimation of important kinetic parameters in copolymerization of ethylene. The kinetic parameters are chosen based on a global sensitivity analysis. The retrospective cost model refinement algorithm is adapted and implemented to estimate the kinetic parameters in real time. The results demonstrate that the estimates of the kinetic parameters converge to theoretical values of the parameters without requiring prior knowledge of the polymerization model or the theoretical parameter values.

Scott et al. [11] revisit five terpolymerization studies and estimate the 'ternary' reactivity ratios. They compare previously determined binary reactivity ratios and newly estimated 'ternary' reactivity ratios for several systems. They discuss the advantages and challenges associated with 'ternary' reactivity ratio estimation.

Garg et al. [12] present a method of uniquely specifying and robustly achieving user-specified product quality in a complex industrial batch process. They demonstrate this method using a lab-scale uni-axial rotational molding process. The method involves data-driven modeling (subspace state-space model) and model predictive control, and is able to reject raw material variation and achieve product quality which is specified through constraints on quality variables. Results from these experimental studies demonstrate the capability of the proposed method in meeting process specifications and rejecting raw material variability.

Bagalkot et al. [13] present case studies on performing polymer rapid tooling inserts and observing different failures over the life of the tool. They identify critical parameters affecting tool life, and the effect of those parameters on different areas of the tool. They categorize modes of the different failures and the underlying mechanisms including the root causes.

Intensified process units such as polymer membrane reactors pose unique challenges pertaining to design and operation that have not been fully addressed. Bishop and Lima [14] present an approach for modeling membrane reactors. Their model allows for the simulation of polymer membrane reactors under nonisothermal and countercurrent operation. This article demonstrates how operability analyses can be used to identify areas of improvement in membrane reactor design.

Topological indices have been computed for various molecular structures. They are numerical invariants associated with molecular structures and are helpful in that they feature many properties. Among these molecular descriptors, the eccentricity connectivity index is of great importance due to its relationship with pharmaceutical properties. Zheng et al. [15] calculate eccentric connectivity, total eccentricity connectivity, augmented eccentric connectivity, first Zagreb eccentricity, modified eccentric connectivity, second Zagreb eccentricity, and the edge version of eccentric connectivity 
indices for the molecular graph of a polyethyleneamidoamine dendrimer. They also calculate explicit representations of the polynomials associated with some of these indices.

Dendrimers are branched organic macromolecules with successive layers of branch units surrounding a central core. The molecular topology and the irregularity of their structure plays a central role in determining structural properties like enthalpy and entropy. Hussain et al. [16] determine irregularity indices that are based on the imbalance of edges, for the molecular graphs associated with some general classes of dendrimers. They also provide graphical analyses of these indices for the dendrimers.

In many applications, knowledge on the irregularity of a molecular structure is of importance. The irregularity provides quantitative information on structure-property relationships and structure-activity relationships as well as various physical and chemical properties. Zhao et al. [17] present a study on the computation and comparison of the irregularity measures of different classes of dendrimers. Their investigation focuses on four irregularity indices; the $\sigma$ irregularity index, the irregularity index by Albertson, the variance of vertex degrees, and the total irregularity index.

Tembely et al. [18] present a mathematical model based on physical principles to simulate droplet impact, spreading, and eventually rebound of a viscoelastic droplet. Their simulations are based on the volume of fluid method in conjunction with a dynamic contact model accounting for the hysteresis between the droplet and substrate. They report that while the kinematic phase of droplet spreading seems to be independent of both the substrate and fluid rheology, the recoiling phase are highly influenced by those operating parameters. Their model can be used to optimize 2D/3D printing of complex fluids.

In PLP, the behavior of the degree of branching and backbiting reactions has not been well-understood yet at high laser frequencies and at relatively high reaction temperatures due to the inherent difficulties in the determination of the degree of branching of polymers. Hamzehlou et al. [19] evaluate the validity of different explanations of the recovery of PLP-molar mass distribution at high laser frequencies using a simulation study. They show that the reduction in the backbiting reaction rate at a high laser frequency, and the consequent decrease in the degree of branching, are not a necessary condition for recovering the PLP-molar mass distribution.

I would like to thank all the contributors for their contributions to this Special Issue, and Processes for supporting this issue.

Conflicts of Interest: The author declares no conflicts of interest.

\section{References}

1. Gooneie, A.; Schuschnigg, S.; Holzer, C. A review of multiscale computational methods in polymeric materials. Polymers 2017, 9, 16. [CrossRef] [PubMed]

2. Askadskiı̌, A.A. Computational Materials Science of Polymers; Cambridge Int Science Publishing: Cambridge, UK, 2003.

3. Zeng, Q.; Yu, A.; Lu, G. Multiscale modeling and simulation of polymer nanocomposites. Prog. Polym. Sci. 2008, 33, 191-269. [CrossRef]

4. Tobita, H. Universal Relationships in Hyperbranched Polymer Architecture for Batch and Continuous Step Growth Polymerization of AB2-Type Monomers. Processes 2019, 7, 220. [CrossRef]

5. Atan, M.F.; Hussain, M.A.; Abbasi, M.R.; Khan, M.J.H.; Fazly Abdul Patah, M. Advances in Mathematical Modeling of Gas-Phase Olefin Polymerization. Processes 2019, 7, 67. [CrossRef]

6. Riazi, H.; Arabi Shamsabadi, A.; Grady, M.C.; Rappe, A.M.; Soroush, M. Method of Moments Applied to Most-Likely High-Temperature Free-Radical Polymerization Reactions. Processes 2019, 7, 656. [CrossRef]

7. Zaccaria, F.; Cipullo, R.; Correa, A.; Budzelaar, P.H.; Busico, V.; Ehm, C. Separating Electronic from Steric Effects in Ethene/ $\alpha$-Olefin Copolymerization: A Case Study on Octahedral [ONNO] Zr-Catalysts. Processes 2019, 7, 384. [CrossRef]

8. Darvishi, A.; Rahimpour, M.R.; Raeissi, S. A Theoretical and Experimental Study for Screening Inhibitors for Styrene Polymerization. Processes 2019, 7, 677. [CrossRef] 
9. Lathrop, P.M.; Duan, Z.; Ling, C.; Elabd, Y.A.; Kravaris, C. Modeling and Observer-Based Monitoring of RAFT Homopolymerization Reactions. Processes 2019, 7, 768. [CrossRef]

10. Salas, S.D.; Brandão, A.L.; Soares, J.B.; Romagnoli, J.A. Data-Driven Estimation of Significant Kinetic Parameters Applied to the Synthesis of Polyolefins. Processes 2019, 7, 309. [CrossRef]

11. Scott, A.J.; Gabriel, V.A.; Dubé, M.A.; Penlidis, A. Making the Most of Parameter Estimation: Terpolymerization Troubleshooting Tips. Processes 2019, 7, 444. [CrossRef]

12. Garg, A.; Abdulhussain, H.A.; Mhaskar, P.; Thompson, M.R. Handling Constraints and Raw Material Variability in Rotomolding through Data-Driven Model Predictive Control. Processes 2019, 7, 610. [CrossRef]

13. Bagalkot, A.; Pons, D.; Symons, D.; Clucas, D. Categorization of Failures in Polymer Rapid Tools Used for Injection Molding. Processes 2019, 7, 17. [CrossRef]

14. Bishop, B.A.; Lima, F.V. Modeling, Simulation, and Operability Analysis of a Nonisothermal, Countercurrent, Polymer Membrane Reactor. Processes 2020, 8, 78. [CrossRef]

15. Zheng, J.; Iqbal, Z.; Fahad, A.; Zafar, A.; Aslam, A.; Qureshi, M.I.; Irfan, R. Some eccentricity-based topological indices and polynomials of poly (EThyleneAmidoAmine)(PETAA) dendrimers. Processes 2019, 7, 433. [CrossRef]

16. Hussain, Z.; Munir, M.; Rafique, S.; Hussnain, T.; Ahmad, H.; Chel Kwun, Y.; Min Kang, S. Imbalance-based irregularity molecular descriptors of nanostar dendrimers. Processes 2019, 7, 517. [CrossRef]

17. Zhao, D.; Iqbal, Z.; Irfan, R.; Chaudhry, M.A.; Ishaq, M.; Jamil, M.K.; Fahad, A. Comparison of Irregularity Indices of Several Dendrimers Structures. Processes 2019, 7, 662. [CrossRef]

18. Tembely, M.; Vadillo, D.; Soucemarianadin, A.; Dolatabadi, A. Numerical Simulations of Polymer Solution Droplet Impact on Surfaces of Different Wettabilities. Processes 2019, 7, 798. [CrossRef]

19. Hamzehlou, S.; Aboudzadeh, M.A.; Reyes, Y. On the Recovery of PLP-Molar Mass Distribution at High Laser Frequencies: A Simulation Study. Processes 2019, 7, 501. [CrossRef]

(C) 2020 by the author. Licensee MDPI, Basel, Switzerland. This article is an open access article distributed under the terms and conditions of the Creative Commons Attribution (CC BY) license (http://creativecommons.org/licenses/by/4.0/). 